\title{
Surface Curvature Effects in the Aqueous Ionic Solvation of the Chloride Ion
}

\author{
Steven J. Stuart* \\ Department of Chemistry, Clemson University, Clemson, South Carolina 29634 \\ B. J. Berne ${ }^{\dagger}$ \\ Department of Chemistry and Center for Biomolecular Simulation, Columbia University, \\ New York, New York 10027
}

Received: May 20, 1999; In Final Form: July 30, 1999

\begin{abstract}
Molecular dynamics simulations of hydrated chloride ions have been performed with a polarizable model for interface geometries of varying curvature, ranging from clusters to planar surfaces. The solvation depth profile of the chloride ion is found to depend dramatically on the curvature of the interface. This behavior is consistent with the structure-breaking character of the chloride ion, and is successfully analyzed in terms of a simple geometric model. This model is expected to be useful in predicting analogous behavior in other ionic species.
\end{abstract}

\section{Introduction}

The aqueous solvation of charged species is a fundamental and interesting topic that has been studied both theoretically and experimentally for decades. In particular, solvation processes at the air-water interface are important in fields ranging from atmospheric chemistry to electrochemistry. Most work to date has been done at planar interfaces, despite the fact that interfaces with nonzero curvature are important in many environmental and experimental systems.

As an attempt to explore the influence of surface curvature on aqueous ionic solvation, this paper will focus on the solvation of the chloride ion near interfaces of varying curvature. The principal issue is whether the chloride is solvated interfacially, i.e., at or near the surface; or internally, with a solvation structure indistinguishable from that in the bulk. The conclusion that has emerged from a variety of experimental studies and computer simulations is that the larger halide ions $\left(\mathrm{Cl}^{-}, \mathrm{Br}^{-}\right.$, and $\left.\mathrm{I}^{-}\right)$are preferentially solvated near curved interfaces, but not at planar interfaces. ${ }^{1-4}$

The conclusions regarding curved interfaces come from the study of small molecular clusters, and provide limited evidence for surface solvation. Experimental techniques which have been used to study these clusters include high-pressure mass spectrometry ${ }^{5,6}$ and photoelectron spectroscopy, ${ }^{7,8}$ each of which provides only indirect evidence of cluster structure. Vibrational predissociation spectroscopy reveals information about hydrogen bonding, and thus more direct evidence for cluster structure. These studies indicate that $\mathrm{Cl}^{-}, \mathrm{Br}^{-}$, and $\mathrm{I}^{-}$are asymmetrically solvated in clusters containing up to 6 water molecules. ${ }^{9-11}$ The fluoride anion, on the other hand, is solvated internally in these small clusters. Larger clusters, with up to 15 water molecules, have been studied by photoelectron spectroscopy. 7,8 These results have also been interpreted to indicate surface solvation for $\mathrm{Br}^{-}$and $\mathrm{I}^{-}$, although the results are not entirely conclusive.

The conclusion that the larger halides are solvated externally in water clusters is supported by computer simulation, as well. Empirical potential models which include a treatment of

\footnotetext{
* Corresponding author. E-mail: ss@ clemson.edu.

†-mail: berne@chem.columbia.edu.
}

molecular polarizability are able to reproduce not only the available experimental data, ${ }^{4,12,13}$ but also the qualitative differences between fluoride and the larger halides. ${ }^{14,15}$ Note that a polarizable solvent model is necessary in these simulations; nonpolarizable models are not as successful in reproducing experimental results, and predict rather different solvation structures from the polarizable models. ${ }^{1,3,16}$

The contribution of the polarizability of the halide ion is less clear. Simulations of small clusters (1-6 waters) have found that including the polarizability of the ion affects the equilibrium between surface and interior solvation states. ${ }^{17}$ Computational studies of larger clusters (20-255 water molecules), on the other hand, have indicated that the ion's polarizability has relatively little effect on the cluster structures and energetics. ${ }^{2,4}$ Recent experimental results indicate that the polarizability of the ion plays a key role in determining the vibrational predissociation spectra of these clusters, and thus presumably contributes significantly to the bonding interactions. ${ }^{18}$ Indeed, these studies suggest that large anionic polarizabilities may be the primary factor leading to surface solvation for nonaqueous solvents such as methanol that can form only a single hydrogen bond. At present, it appears that the use of a polarizable ion is the more prudent alternative.

For the chloride ion in particular, molecular dynamics studies with fully polarizable models have indicated that the ion remains solvated interfacially in clusters of up to 20 water molecules, ${ }^{1-4}$ in agreement with the available experimental data. ${ }^{8,9}$ Somewhat surprising, however, is the simulation result that interfacial solvation persists in clusters containing as many as 255 water molecules. ${ }^{4}$ These results, while intriguing, have no experimental confirmation.

At planar interfaces, the situation is reversed. Experimental measurements of the surface tension and surface potential indicate that the halide ions are repelled from the interface, maintaining one or more complete solvation shells at all times. ${ }^{19}$ Previous nonpolarizable simulations agree with these results. ${ }^{20}$ It has been assumed that the more complete polarizable models will also reproduce this experimental behavior, although that assumption has not previously been tested. This interesting behavior of chloride and the larger halide ions, in which the 
solvation structure depends on the curvature of the interface, has not been explained adequately to date.

The present investigation represents a detailed analysis of the solvation behavior of $\mathrm{Cl}^{-}$at both planar and curved interfaces. The purpose of this study was 2-fold. The first goal was to determine whether a polarizable potential model, which successfully reproduces experimental results for small clusters and bulk solution, also performs reliably at planar interfaces. This will help to determine the reliability of predictions regarding large, bulklike clusters which have not been studied experimentally. The second goal was to understand the driving force behind curvature-dependent solvation behavior.

The following section provides a description of the simulation model used, and Section III summarizes the simulation techniques. The results from simulations of both curved and planar interfaces of various sizes are reported in Section IV. A simple solvation model is developed in Section $\mathrm{V}$ that accounts for the observed behavior and allows predictions to be made regarding the extent of surface solvation in systems of arbitrary curvature. The results and analysis are summarized in Section VI.

\section{Computational Method}

The potential model used for the water molecules is a modification of the TIP4P water model, ${ }^{21}$ extended to use fluctuating partial charges. This fluctuating-charge TIP4P model, referred to as TIP4P-FQ, or the fluc- $q$ model, has been described in detail elsewhere. ${ }^{4,22}$ Briefly, this model allows the values of the partial charges assigned to each atom to fluctuate dynamically as the simulation proceeds, as a means of modeling the polarizability of the water molecule. The dynamics of the partial charges are modeled by using an extended Lagrangian technique to propagate the charge variables through time as additional dynamic variables. The charges are assigned a fictitious inertial mass, $\mu_{q}$, which is extremely light, allowing the charges to rapidly adjust to the changes in the electrostatic potential produced by the more slowly moving nuclei.

The interactions in the system can be divided into singlecharge self-energies and pairwise interactions

$$
V=\sum_{i} V_{i}^{\text {self }}+\frac{1}{2} \sum_{i} \sum_{j \neq i} V_{i j}^{\text {Coul }}+\frac{1}{2} \sum_{i} \sum_{j \neq i} V_{i j}^{\mathrm{LJ}}
$$

The self-energy for a charge site $i$

$$
V_{i}^{\mathrm{self}}=\chi_{i}^{\circ} q_{i}+\frac{1}{2} J_{i i}^{\circ} q_{i}^{2}
$$

arises from the theory of electronegativity equalization, ${ }^{23}$ and describes the energy associated with changing the value of the partial charge assigned to a particular site. The Coulombic pair energy

$$
V_{i j}^{\text {Coul }}=q_{i} q_{j} J_{i j}\left(r_{i j}\right)
$$

specifies the electrostatic interaction between partial charges $q_{i}$ and $q_{j}$ separated by a distance $r_{i j}$. The partial charges are not point charges, but have the spatial distribution of a Slater $s$ orbital. Since these charges are delocalized, the Coulomb interaction $J_{i j}\left(r_{i j}\right)$ is evaluated using a Coulomb integral, rather than the $1 / r_{i j}$ interaction usually used for point charges. This is particularly important for intramolecular Coulomb interactions; at intermolecular distances, the $J(r)$ Coulomb interaction is approximated by $1 / r$ to save computer time. The Lennard-Jones (LJ) pair energy is given by the familiar 12-6 interaction between all $\mathrm{LJ}$ sites,

$$
V_{i j}^{\mathrm{LJ}}=4 \epsilon_{i j}\left[\left(\frac{\sigma_{i j}}{r_{i j}}\right)^{12}-\left(\frac{\sigma_{i j}}{r_{i j}}\right)^{6}\right]
$$

The values of the $\sigma_{i j}, \epsilon_{i j}, \chi_{i}^{\circ}$, and $J_{i i}^{\circ}$ parameters, along with all other information needed to implement the potential, are provided in ref 22 .

The model used for the chloride ion is the polarizable Drude ion model developed for earlier studies of aqueous chloride clusters. ${ }^{4}$ In this model, a Drude oscillator ${ }^{24,25}$ with a net charge of $-1|e|$ interacts with the other particles in the system via Coulomb and Lennard-Jones interactions. A nonzero displacement of the Drude oscillator results in a dipole moment for the chloride ion. The ion model parameters are chosen so that the chloride ion polarizability matches the experimental value. The mass distribution within the Drude ion is chosen so that the spring can adjust much more quickly than the nuclear coordinates to changes in the local electric field. Both the fluc- $q$ and Drude models allow a Born-Oppenheimer-like separation of time scales between the electrostatic degrees of freedom and the nuclear motion. The complete set of parameters describing the chloride Drude ion model is contained in ref 4.

Traditional molecular dynamics simulations of liquid water typically use time steps of approximately $\Delta t=1 \mathrm{fs}$. This time step is appropriate for both the atomic coordinates and fluctuating charges in the TIP4P-FQ model, which is only marginally more computationally expensive than a fixed-charge model. The extremely high characteristic frequency of the Drude ion, however, requires a time step four times smaller, $\delta t=0.25 \mathrm{fs}$. Rather than integrating the dynamics of the entire system with this smaller time step, the reversible reference system propagator algorithm (rRESPA) is used to integrate the dynamics with multiple time scales. ${ }^{26}$ A particle-based split is used in the rRESPA decomposition of the Liouvillian, ${ }^{27}$ so that the quickly oscillating Drude ion degrees of freedom are advanced with a time step of $\delta t=0.25$ fs and the more slowly moving water molecules are advanced with $\Delta t=1 \mathrm{fs}$.

\section{Simulation Details}

A. Spherical Clusters. Molecular dynamics simulations of $\mathrm{Cl}\left(\mathrm{H}_{2} \mathrm{O}\right)_{n}{ }^{-}$clusters containing between 1 and 255 water molecules have been reported previously. ${ }^{4}$ The results for the $n=100$ and 255 clusters are presented here for comparison with the results at a planar interface. These clusters were simulated in a vacuum, with no periodic boundary conditions applied. The simulations were performed in the microcanonical ensemble with an average kinetic energy corresponding to a macroscopic temperature of $298 \mathrm{~K}$. The statistics were collected over a minimum of 300 ps after an initial equilibration period. Occasionally, water molecules would leave the cluster and due to the absence of periodic boundary conditions would typically not recondense. In no case did more than $5 \%$ of the cluster evaporate over the length of the simulation, however. The temperature was lowered by a total amount of less than $5 \mathrm{~K}$ by these evaporation events.

B. Planar Interface. Simulations were also performed for chloride ions at the planar water-vapor interface, for systems of various sizes. These systems consisted of a chloride ion solvated in lamellar slabs of either 255 or 499 water molecules, periodically replicated in all three dimensions. For each different-sized system, bulklike molecular volumes of $30.0 \AA^{3}$ for water and $85.5 \AA^{3}$ for the chloride ion were used to estimate the volume of the solution phase, $V=L^{3}$. The simulation unit 


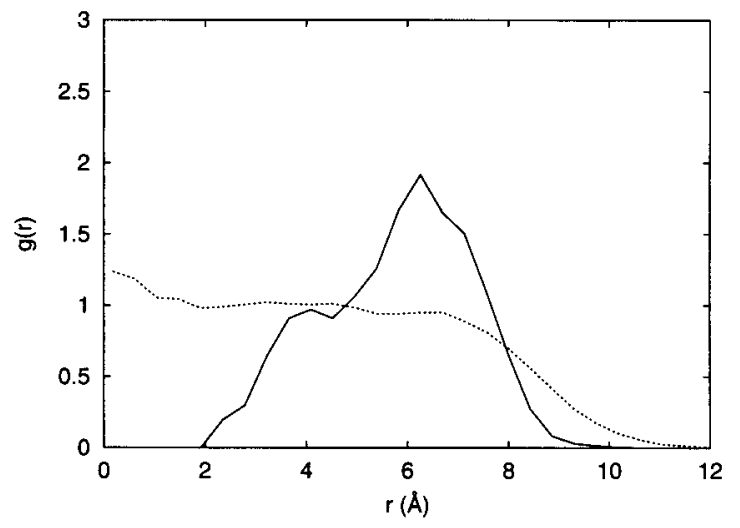

Figure 1. Radial distribution function for the distance between the chloride ion and the center of mass of the $\mathrm{Cl}\left(\mathrm{H}_{2} \mathrm{O}\right)_{100}^{-}$cluster (solid line). The size of the cluster is indicated by the distribution of distances between oxygen atoms and the center of mass (dashed line). The oxygen radial distribution function is scaled relative to the density in bulk water at $298 \mathrm{~K}$. The chloride curve is scaled arbitrarily.

cell was then chosen to be of dimensions $L \times L \times 3 L$. The imposition of periodic boundary conditions then resuled in lamellae of approximate width $L$, separated by vacuum layers of width $2 L$. The interface remained planar, minimizing the surface energy of the system. For the 256-molecule system, $L$ $=19.78 \AA$, and for the 500-molecule system, $L=24.69 \AA$.

These simulations were also performed in the microcanonical ensemble at conditions corresponding to a temperature of 298 $\mathrm{K}$. As with the cluster simulations, water molecules were free to evaporate from the interface. Because of the periodic boundary conditions imposed in these simulations, however, the gaseous water molecules typically reentered the liquid after some time in the vapor phase.

The long-range electrostatic interactions were evaluated using the three-dimensional Ewald sum. ${ }^{28,29}$ Various implementations of a two-dimensional Ewald sum exist, ${ }^{30,31}$ but these algorithms can require large numbers of $k$ vectors and increased computation time when electric fields are present at the interface.

\section{Results}

A. Spherical Clusters. As observed previously, ${ }^{4}$ the chloride ion is preferentially solvated toward the exterior of the spherical water clusters when $n \leq 255$. This is visible in the radial density distributions for the chloride ion, which are displayed in Figure 1 for the 100-water cluster and Figure 2 for the 255-water cluster. These graphs represent the relative $\mathrm{Cl}^{-}$density as a function of distance from the center of mass of the cluster, scaled with the appropriate factor of $4 \pi r^{2}$ to account for the increased volume at larger distances. Similar radial density distributions are plotted for the water molecules, as an indication of the size of the clusters.

If the surface of the cluster is defined by the radial distance beyond which the average solvent density is less than half of its value in the bulk, then the 100-water cluster is seen to be approximately $9 \AA$ in radius. The chloride ion density is highest between 4 and $8 \AA$ from the center of the cluster, corresponding to a depth of $1-5 \AA$ below the cluster's surface. The ion is rarely found at depths of more than $6 \AA$ beneath the surface, and almost never penetrates to the center of the cluster. These results are independent of the initial conditions. When the simulation was started with the ion at the center of the cluster, the ion diffused to the interface within tens of picoseconds, and did not return to the center for the remainder of the simulation.

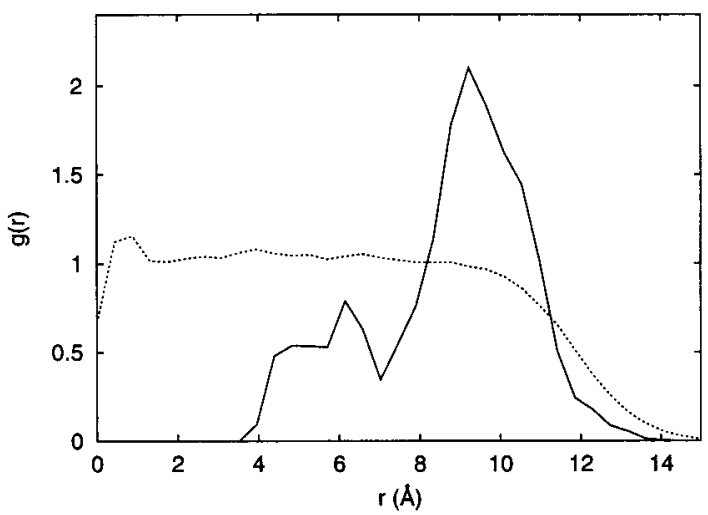

Figure 2. Radical distribution function for the distance between the chloride ion and the center mass of the $\mathrm{Cl}\left(\mathrm{H}_{2} \mathrm{O}\right)_{255}^{-}$cluster (solid line). The size of the cluster is indicated by the distribution of distances between oxygen atoms and the center of mass (dashed line). The oxygen radial distribution function is scaled relative to the density in bulk water at $298 \mathrm{~K}$. The chloride curve is scaled arbitrarily.

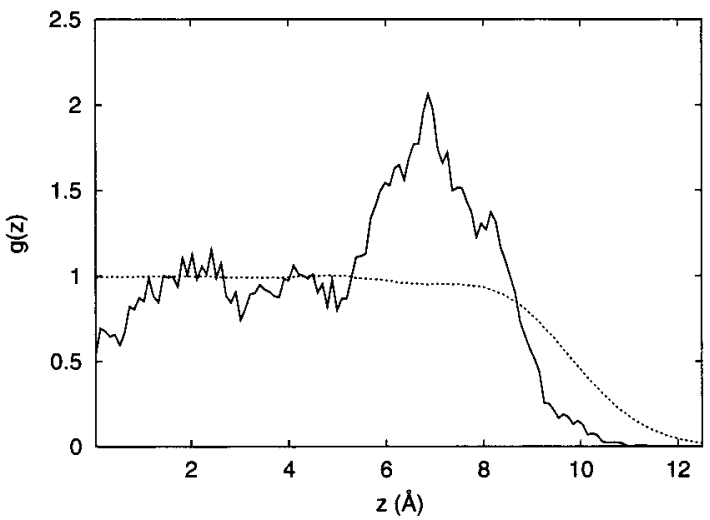

Figure 3. Distribution function for the distance between a Drude chloride ion and the center of a $20-\AA$ lamellar slab (solid line). The location of the interface is indicated by the distribution of distances between oxygen atoms and the center of the lamella (dashed line). The distribution function for oxygen is scaled relative to the density in bulk water at $298 \mathrm{~K}$. The chloride curve is scaled arbitrarily.

The data presented in Figure 1 are an average of the results from 700 ps of simulation time with two different initial conditions.

The situation is even clearer for the 255-water cluster, as shown in Figure 2. Again, the chloride ion concentration is largely concentrated at a depth of $1-5 \AA$ beneath the surface, and is negligible at depths greater than $8 \AA$. The chloride is restricted to the outer one or two molecular solvent layers, and never penetrates to the center of the cluster. Once again, the radial distributions displayed in Figure 2 are an average over simulations with two independent starting conditions, representing a total of $600 \mathrm{ps}$ of simulation.

B. Planar Interface. When a chloride ion is solvated at a planar interface, the solvation structure is quite different. Figure 3 shows the average density of chloride ions as a function of distance from the center of the lamella for the system with 255 water molecules. Here the data have been averaged not only over two different initial conditions (chloride at the surface and chloride at the center), but also over the two equivalent interfaces of the lamella. The results for both interfaces were observed to be qualitatively similar before being combined; in all simulations presented here, the ion visited both interfaces over the course of the simulation. Although the ion demonstrates a slight preference for solvation within $1-4 \AA$ of the interface, it is not 


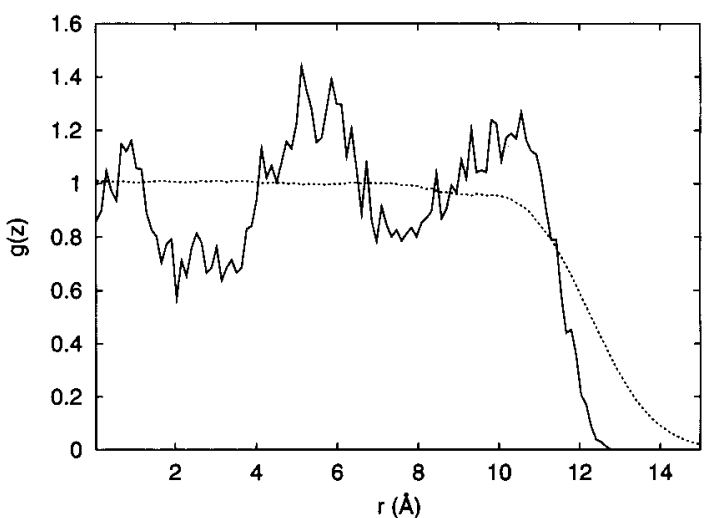

Figure 4. Solvation depth distribution function for the distance between a chloride ion and the center of a $24-\AA$ lamellar slab (solid line). The location of the interface is indicated by the distribution of distances between oxygen atoms and the center of the lamella (dashed line). The distribution function for oxygen is scaled relative to the density in bulk water at $298 \mathrm{~K}$. The chloride curve is scaled arbitrarily.

excluded from the bulklike core of the system, as was the case for the spherical clusters.

For the larger system, with 499 water molecules, the results are similar, as shown in Figure 4. The chloride ion ventures to within $1 \AA$ of the edge of the slab, and also penetrates to the interior of the cluster. The chloride ion samples the entire volume of the lamalla over the course of the 300-ps simulation, although there is some indication of interface-induced structure at depths of approximately 2 and $7 \AA$ beneath the interface. Unfortunately, the data are not sufficiently averaged after 600 ps of simulation time to allow us to draw definitive conclusions about layering at the interface.

While the statistical distribution of the ion density indicates that it approaches to within $1 \AA$ of the average interface position, detailed investigation of the simulation trajectories indicated that the ion always maintained a full solvation shell. On the occasions when the ion approached the average position of the interface, a bulge would form on the surface to accommodate the first solvation shell. The average coordination number of the chloride ion in these simulations was $6.1 \AA$, identical to the value in bulk solution. ${ }^{4}$ It would be interesting to examine the distribution of depths beneath the instantaneous surface, rather than the distribution beneath the average location of the interface. Unfortunately, however, the instantaneous surface is a difficult quantity to define.

These results are an important validation of the TIP4P-FQ model. Experimentally, the increase in surface tension with salt concentration can be used to determine the extent to which ions are repelled from a planar interface. ${ }^{32}$ For alkali halide solutions, surface tension data are consistent with approximately one monolayer of water at the surface that is entirely ion-free, with a Boltzmann distribution of ions further from the surface. ${ }^{19}$ This indicates that the ions always maintain a full solvation shell beneath the surface. The simulation results are in complete accord with this experimental interpretation.

The simulation result that the solvation structure is different beneath the surfaces of clusters and flat interfaces reveals that there is in fact a surface solvation effect that depends solely on the curvature of the interface. This is the first study that has used the same model to study anionic solvation at both curved and flat surfaces, removing the possibility that previously observed surface curvature effects could be due to differences in potential models.

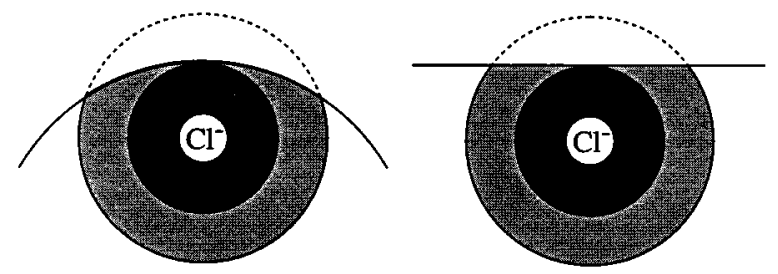

Figure 5. Model of the surface solvation effect: The chloride ion has strongly favorable interactions with the closest water molecules, but the net interaction with more distant molecules is much less favorable. More of the energetically unfavorable shell can be excluded from the liquid phase at a curved interface than at a planar interface.

\section{Analysis}

It is of interest to analyze the computer simulation results in greater detail, to determine the reason for the curvaturedependent solvation behavior. In particular, it would be useful to determine if the structure-breaking character of the chloride ion is responsible for this phenomenon, as has been asserted previously. ${ }^{4}$

Characterization of an ion as structure-making or structurebreaking is a convenient shorthand for describing the influence of the ion on the structure of the surrounding solvent. A structure-breaking ion can be viewed as disrupting the hydrogenbonding network in the liquid to a greater extent than is compensated by the ordering influence of the ion on the first and second solvation shells. This effect manifests itself in many ways, but is perhaps most clearly conceptualized as a positive structural contribution to the hydration enthalpy. ${ }^{33}$

For our current purposes, however, it will be instructive to consider the solvation of the chloride ion in terms of an energy balance. The strongly favorable chloride-water bonds come with an associated penalty: every water molecule in the first solvation shell of the chloride ion must have sacrificed one or more water-water hydrogen bonds in order to interact favorably with the chloride ion. The chloride-water interactions of approximately $15 \mathrm{kcal} / \mathrm{mol}^{35}$ are stronger than the $\sim 5 \mathrm{kcal} / \mathrm{mol}^{35}$ water-water interactions. This is responsible for the strongly negative solvation energy of the ion. According to the argument for structure-breaking solvation, however, the water molecules in the second solvation shell will be participating in fewer hydrogen bonds than if they were in the bulk. ${ }^{36}$ For the secondshell water molecules, the interaction with the chloride ion will not necessarily be favorable enough to compensate for the loss of hydrogen bonds, and water molecules in this shell can be energetically destabilized, relative to molecules in the bulk.

This reasoning can be used to explain the energetic benefit due to solvation of a chloride ion just beneath the surface of a cluster. At certain distances below the interface, the low-energy first solvation shell will be fully populated, but the destabilized second shell will be only partially filled. Whether or not this surface solvation will occur will depend on the delicate balance among stabilization of the first solvation shell, destabilization of the second shell, and interactions at greater distances. It is clear from Figure 5, however, that any tendency toward interfacial solvation-if present-will be enhanced at curved interfaces, where a greater fraction of the second solvation shell can be excluded from the cluster.

We have tested this hypothesis by constructing a simple model for solvation beneath an interface of arbitrary curvature. In this model, the solvation energy of a solvent molecule is assumed to depend only on its distance from the ion. This distance-dependent average solvation energy can be estimated from simulations of the bulk solution, in the absence of any 
perturbing interface. Then in order to estimate the energy associated with solvating an ion at a particular depth beneath a given interface, the solvation energy expression can be integrated over the volume of interest.

The first step in this procedure is to obtain average solvation energies for water molecules as a function of their distance from the chloride ion, using the TIP4P-FQ model. Since the TIP4PFQ potential contains many-body terms, there are some ambiguities involved in decomposing the system energy into pairwise interactions. Thus we will be explicit with all definitions. The potential energy of the system is a sum of pair energies $V_{i j}{ }^{\mathrm{Coul}}$ and $V_{i j}^{\mathrm{LJ}}$, and self-polarization energies, $V_{i}^{\text {self }}$, as shown in eq 1 . We now define a pair interaction energy $E_{i j}$ between sites $i$ and $j$, such that

$$
E_{i j}=V_{i j}^{\text {Coul }}+V_{i j}^{\mathrm{LJ}}+\frac{V_{i j}^{\text {Coul }}}{\sum_{k \neq j} V_{i k}^{\text {Coul }}} V_{i}^{\text {self }}+\frac{V_{i j}^{\text {Coul }}}{\sum_{k \neq j} V_{k j}^{\text {Coul }}} V_{j}^{\text {self }}
$$

With this definition, the self-energies are distributed among the various pairwise interactions in proportion to the electrostatic interactions that give rise to the polarization response. The exact division of self-energies into pairwise terms is arbitrary, and other definitions are possible. Defining a pair energy $E_{i j}$ in this way allows us to write

$$
V=\frac{1}{2} \sum_{i} \sum_{j \neq i} E_{i j}
$$

expressing the potential energy formally in a pairwise form. This is important because each contribution to the system energy can now be assigned an interaction distance $r_{i j}$, including the self-energies.

We can further identify the energy associated with a particular site $i$ to be

$$
E_{i}=\frac{1}{2} \sum_{j \neq i} E_{i j}
$$

so that we have

$$
\sum_{i} E_{i}=V
$$

Although it is by no means a unique method of partitioning the total many-body system energy into contributions from individual interaction sites, this definition of a site energy is nonetheless intuitively appealing.

The molecular energy $E_{I}$ for a molecule $I$ can be obtained by summing the individual site energies $E_{i}$ for all sites $i$ within molecule $I$. A mean solvent energy $E_{\mathrm{H}_{2} \mathrm{O}}=\left\langle E_{I}\right\rangle$ can be obtained by averaging the molecular energies of an ensemble of solvent molecules over the course of a simulation. (Note that because the definitions above attribute only half of the interaction energy $E_{i j}$ to the molecule containing site $i$, the molecular energy $E_{I}$ and solvent energy $E_{\mathrm{H}_{2} \mathrm{O}}$ differ from the usual solvation energy by a factor of 2.)

We are particularly interested in describing the mean energy of a solvent molecule, in the presence of the solute, as a function of the distance $r$ between the solvent molecule and the solute

$$
E_{\mathrm{H}_{2} \mathrm{O}}(r) 4 \pi r^{2} \mathrm{~d} r=\frac{1}{\rho}\left\langle\int_{r}^{r+\mathrm{d} r} \sum_{I} E_{I} \delta\left(r_{I}-r^{\prime}\right) \mathrm{d} r^{\prime}\right\rangle
$$

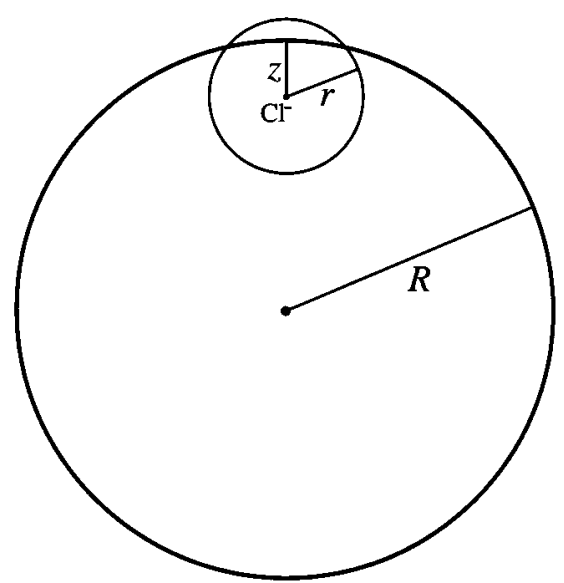

Figure 6. Schematic indicating a chloride ion solvated at a depth $z$ beneath the surface in a cluster of radius $R$. The integral over the volume of the cluster will be performed using shells of radius $r$.

where $\rho$ represents the number density of the solvent molecules and $r_{I}$ is the distance between solvent molecule $I$ and the ion. In the simulations performed here, the position of the solvent molecule was taken to be the location of the oxygen site in the TIP4P-FQ model.

Another definition that will be useful is the excess energy of the solvent

$$
\Delta E(r)=E_{\mathrm{H}_{2} \mathrm{O}}(r)-\langle E\rangle_{\text {bulk }}
$$

where $\langle E\rangle_{\text {bulk }}=\left\langle E_{I}\right\rangle$ is the average solvent energy in the absence of solute, which is $-9.9 \mathrm{kcal} / \mathrm{mol}$ for the TIP4P-FQ model at $298 \mathrm{~K}$ and $1 \mathrm{~g} / \mathrm{cm}^{3}$ (ref 22). There is no distance dependence to $\langle E\rangle_{\text {bulk }}$, since the bulk solvent is homogeneous.

If we integrate the excess solvent energy density $\rho \Delta E(r)$ over the volume of the system, we recover the solvent contribution to the total energy change on solvation of the solute ion. The remaining contribution to the total solvation energy is simply the site energy of the ion itself. Recalling that the solvation energy is twice the ion's site energy, we have

$$
2 E_{\mathrm{Cl}^{-}}=E_{\mathrm{Cl}^{-}}+\rho \int_{V} \Delta E(r) d^{3} r
$$

which allows us to express the solute's site energy in terms of the excess solvent energy. For an infinite bulk system

$$
E_{\mathrm{Cl}^{-}}=\rho \int_{r=0}^{\infty} \Delta E(r) 4 \pi r^{2} \mathrm{~d} r
$$

If the solvent geometry is a spherical cluster with radius $R$, and the solute ion is solvated at a depth $z$ beneath the interface, as depicted in Figure 6, then the integral can be rewritten as

$$
E_{\mathrm{Cl}^{-}}(z)=\rho \int_{r=0}^{\infty} \Delta E(r) A(r ; z, R) \mathrm{d} r
$$

where $A(r ; z, R) \neq 4 \pi r^{2}$ for spherical shells that extend beyond the boundary of the cluster (see Figure 5). In particular, we can write the surface area of the radius- $r$ integration sphere that is contained within the radius- $R$ cluster as

$$
\begin{aligned}
& A(r ; z, R)= \\
& \begin{cases}4 \pi r^{2}, & r \leq z \\
\pi \frac{r}{R-z}\left[R^{2}-(R-z-r)^{2}\right], & z \leq r \leq 2 R-z \\
0, & 2 R-z \leq r\end{cases}
\end{aligned}
$$




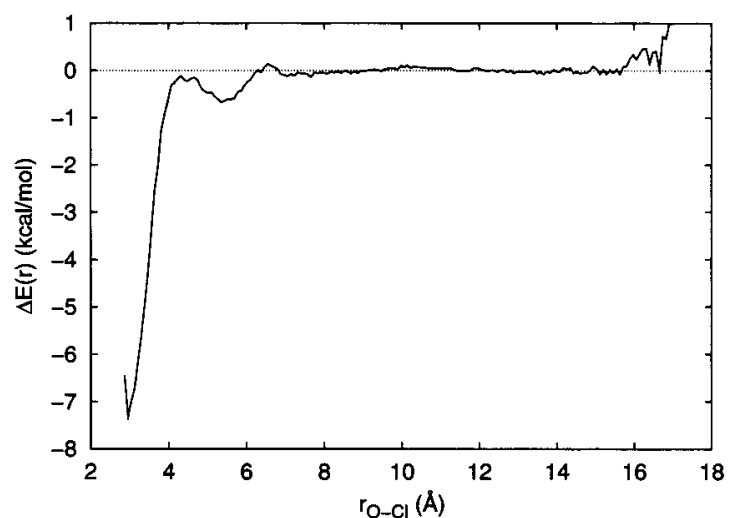

Figure 7. Distance-dependent solvation energy $\Delta E(r)$ for TIP4P-FQ water molecules at a specified distance from a Drude chloride ion in bulk solution, expressed relative to the asymptotic value at large $r$.

A semi-infinite planar interface is equivalent to a cluster of infinite radius, so for the lamellar systems we use

$$
\lim _{R \rightarrow \infty} A(r ; z, R)= \begin{cases}4 \pi r^{2}, & r \leq z \\ 2 \pi r(r+z), & z \leq r\end{cases}
$$

Note that although the use of $A(r ; z, R)$ seems to imply a uniform distribution of solvent molecules, some information about the structure and molecular nature of the solvent is contained in eq 13 in the energy density $\rho E_{\mathrm{H}_{2} \mathrm{O}}$.

Using eqs 13 and 14, we can estimate the energy associated with solvating a chloride ion at a depth $z$ beneath the interface of any spherical cluster of radius $R$ as follows. From a simulation of neat liquid TIP4P-FQ water at $298 \mathrm{~K}$ and $1 \mathrm{~g} / \mathrm{cm}^{3}$, using Ewald sums to calculate the Coulomb interactions, we obtain $\langle E\rangle_{\text {bulk }}=-9.85 \mathrm{kcal} / \mathrm{mol}$. Then, from a simulation of a single Drude chloride ion dissolved in 255 TIP4P-FQ water molecules, again with Ewald boundary conditions, we obtain $\Delta E(r)$ as detailed in eq 10. This function, which amounts to a radial partitioning of the excess solvation energy, was obtained from $100 \mathrm{ps}$ of simulation at $298 \mathrm{~K}$ and a system density of 0.994 $\mathrm{g} / \mathrm{cm}^{3}$, and is plotted in Figure 7. Notice that this calculation of $\Delta E(r)$ only partially supports the simple model illustrated in Figure 5. Water molecules within $3.5 \AA$ of a chloride ion are seen to have energies that are several $\mathrm{kcal} / \mathrm{mol}$ more negative than the bulk average of $-9.85 \mathrm{kcal} / \mathrm{mol}$. This intermolecular distance coincides with the first peak in a $g_{\mathrm{Cl}-\mathrm{O}}$ pair correlation function, ${ }^{4}$ demonstrating that the first shell of water molecules around the chloride ion is very favorably solvated. The water molecules in the second solvation shell, roughly $4.5-5.5 \AA$ from the chloride ion, are also more favorably solvated than water molecules in the bulk. At intermediate distances, 3.5-4.5 from the chloride ion, the solvent molecules have somewhat higher energies, but are still more favorably solvated than in the bulk. These distances correspond to the less densely populated interstitial region between the two solvation shells. ${ }^{37}$ The only water molecules that are energetically destabilized relative to the bulk lie in a narrow region just beyond the second solvation shell ( $6.5 \AA$ from the chloride ion) and a broad region between 9.5 and $11 \AA$ of solvent molecules that are destabilized very slightly, but with statistical significance. (The positive values at $r>15 \AA$ are statistical fluctuations, resulting from the relatively few molecule pairs observed at separations of greater than $L / 2$. When these data are used below to predict solvation energies, $\Delta E(r)$ is set to zero for $r \geq 13.6$.) The general picture derived by the structure-breaking hypothesis is thus qualitatively correct, but with several different regions conceivably providing the impetus for surface solvation.

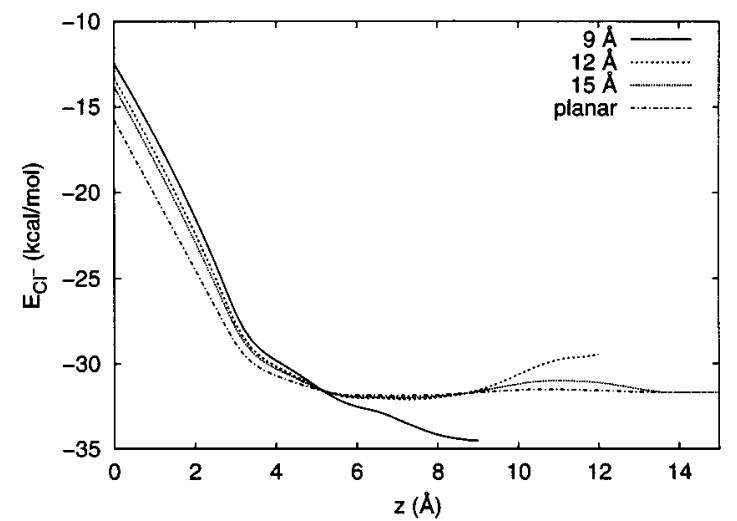

Figure 8. Solvation energy profile for a chloride ion located beneath interfaces of various curvatures. Curves shown are for clusters of radius $9 \AA$ (solid line), $12 \AA$ (dashed line), and $15 \AA$ (dotted line) and for a planar interface (dot-dashed line).

Once the function $\Delta E(r)$ has been obtained from a bulk solution simulation, it can be used to determine approximate solvation energies in clusters of any size and shape. This requires only a simple numerical integration as described by eq 13 , rather than a lengthy molecular dynamics simulation. The results of several such calculations are shown in Figure 8. (Note that since water molecules rarely venture to within $2.9 \AA$ of the chloride ion, $\Delta E(r)$ is not available at $r \leq 2.9 \AA$. For this reason, the lower integration limit in eq 13 was taken to be $2.9 \AA$, rather than zero.) As an example, consider the cluster of radius $12 \AA$ (dashed curve in Figure 8)-roughly the same size as the 255water cluster whose solvation structure is pictured in Figure 2. The figure shows that, according to the simple solvation model, the most energetically favorable solvation depths are between 5 and $9 \AA$ beneath the surface. There is a barrier of several $\mathrm{kcal} / \mathrm{mol}$ associated with penetration to the center of the cluster, and a larger barrier to solvation at the extreme edge of the cluster. Note that the solvation energies within several Angstroms of the edge of the cluster are not reliable, because the bulk-averaged model is incapable of accounting for any modifications in solvent structure near the interface. In particular, simulations indicate that the chloride ion maintains a full solvation shell when approaching the interface-behavior which will not be captured by the model for solvation depths smaller than about $3 \AA$.

The favorable solvation at 5-9 ^ persists in larger clusters, although solvation at larger depths becomes equally favorable, and the barrier separating the subsurface solvation and interior solvation becomes smaller as the curvature of the surface decreases. For a planar interface, the solvation energy is nearly independent of solvation depth below $5 \AA$. Each of these observations is consistent with the solvation structure evident in Figures 2 through 4 . The $9 \AA$ cluster, approximately equivalent to a 100-water cluster, does not indicate a tendency for surface solvation under this simple model, although surface solvation is clearly indicated in Figure 1. We conclude that the solvent in clusters of 100 molecules or less does not behave sufficiently like bulk solution for this model to provide accurate results. (In other words, surface effects in small clusters cause $\Delta E(r)$ to differ appreciably from Figure 7.)

To make more direct comparisons with Figures 1-4, we calculate a simulated $g(z)$ using

$$
g(z) \mathrm{d} z=\frac{\int_{z}^{z+\mathrm{d} z} \mathrm{e}^{-\beta E_{\mathrm{Cl}^{-}\left(z^{\prime}\right)}} \mathrm{d} z^{\prime}}{\int_{0}^{R} \mathrm{e}^{-\beta E_{\mathrm{Cl}}-\left(z^{\prime}\right)} \mathrm{d} z^{\prime}}
$$




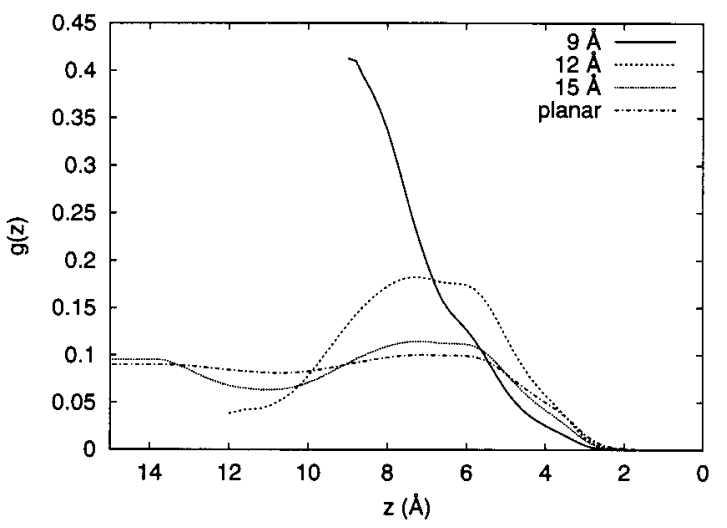

Figure 9. Solvation depth distribution function for a chloride ion solvated by TIP4P-FQ water molecules, located beneath interfaces of various curvatures. Curves shown are for clusters of radius $9 \AA$ (solid line), $12 \AA$ (dashed line), and $15 \AA$ (dotted line) and for a lamellar slab of thickness $30 \AA$ (dot-dashed line). The solvation depth axis is reversed to assist comparison with Figures 1-4.

Note that an accurate prediction of solvation depth should properly account for entropic as well as energetic effects. The largest entropic term would be the $4 \pi(R-z)^{2}$ term that reflects the increased volume accessible to the ion at large radii. This weighting factor is equivalent to the $4 \pi r^{2}$ term used to normalize a traditional pair correlation function. Thus Figures 1-4 and eq 16 both neglect this term intentionally. There are more subtle entropic effects associated with the restriction of solvent conformation space upon the association of chloride-water bonding pairs. No attempt has been made to account for these effects.

Solvation depth distribution functions have been calculated using eq 16 for various cluster sizes, and are presented in Figure 9. These figures reinforce the conclusions that were drawn from the solvation energy profiles. For the $12 \AA$ cluster, which is comparable in size to the 255-water cluster of Figure 2, subsurface solvation is most probable. Interior solvation becomes more likely as the surface curvature decreases, however. The $15 \AA$ cluster, roughly equivalent to a 500-water cluster, is the first for which interior solvation is as probable as subsurface solvation. Significantly, the simplicity of the geometric model allows this prediction to be made without requiring the simulation of an actual 500-water cluster. And for a planar interface, Figure 9 demonstrates that the ion density is almost uniform beneath the surface, with minor oscillations which are due to the structure in $\Delta E(r)$. Aside from the $9 \AA$ cluster, then, these curves are very reminiscent of Figures 2 through 4 . The precise depth of the subsurface solvation layer differs by several Angstroms from the simulation results, however. This is not surprising, and is due to a combination of factors, including some ambiguity in defining the exact position of the surface, the overly sharp transition from liquid phase to vacuum implied in eq 14, and an inability to account for any structuring of the interface by the chloride ion. This last factor is most likely quite important, since simulation trajectories show a distinct tendency for the chloride ion to induce a bulge of tightly coordinated water molecules at the interface when solvated very near to the surface of both clusters and lamellae. These differences notwithstanding, the simple geometric solvation model indicates that a simple model based on distance-dependent solvation energies is sufficient to describe the basic phenomenon of surface solvation. The chloride ion remains near the interface when there is some energetic advantage to leaving certain of the chloride's solvation shells only partially populated. This

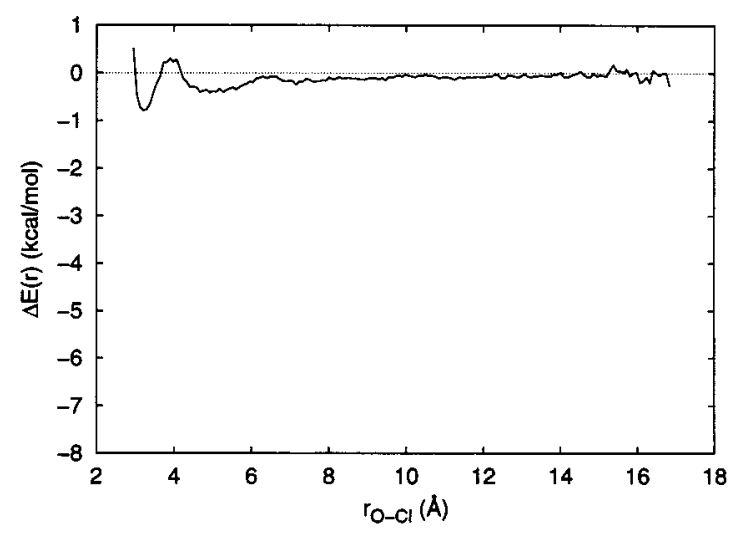

Figure 10. Distance-dependent solvation energy $\Delta E(r)$ for nonpolarizable TIP4P water molecules at a specified distance from an OPLS chloride ion in bulk solution, expressed relative to the asymptotic value at large $r$.

advantage decreases as the interface becomes more planar, leading to less pronounced surface solvation at planar interfaces.

Previous studies have found that nonpolarizable models do not exhibit this surface solvation effect for the chloride ion. ${ }^{1,3,4}$ It is thus of interest to use the simple model described above to determine the reason for this difference between polarizable and nonpolarizable models. The distance-dependent solvation energy $\Delta E(r)$ is shown for the nonpolarizable OPLS model ${ }^{21,38}$ in Figure 10. The most obvious difference from the equivalent curve for the polarizable model shown in Figure 7 is that the first shell of water molecules is much less strongly solvated. This is due to the inability of the solvent water molecules to increase their dipole moment in response to a local electric field in the nonpolarizable OPLS model. Earlier studies have observed similar effects, noting that an enhanced dipole moment on the water molecules is an important factor responsible for the surface solvation effect. ${ }^{4}$ Although the chloride ion solvation energy is similar for both polarizable and nonpolarizable models, ${ }^{4}$ a greater fraction of this solvation energy arises from the first solvation shell in the polarizable model; more distant solvent molecules contribute little, and some act to reduce the solvation energy. For the nonpolarizable model, on the other hand, a large fraction of the total solvation energy results from interactions with solvent molecules beyond the first solvation shell. Figure 10 shows that the solvent contribution remains negative out to distances of about $15 \AA$, approaching the bulk value slowly and nearly monotonically. Thus there is no energetic benefit to the surface solvation of the ion when simulated with this model. This is verified in Figure 11, which shows the solvation depth distribution for the same systems considered in Figure 9. The geometric solvation model shows no evidence for surface solvation in any of the spherical clusters, which is in agreement with the simulation results. ${ }^{4}$ In every case, the chloride ion density is highest at the center of the cluster.

\section{Discussion}

We have found that the surface solvation that is evident for the chloride ion in aqueous clusters containing up to 255 water molecules is not present at planar interfaces, in agreement with available experimental results. By using a simple geometric model to provide insight into the solvation beneath interfaces of various curvatures, it was confirmed that the surface solvation is primarily due to an energetic benefit associated with reducing the occupation of certain solvation shells of the ionic species. Furthermore, this model is able to make a prediction about the cluster size ( $~ 500$ molecules) above which the ion will begin to exhibit interior solvation. 


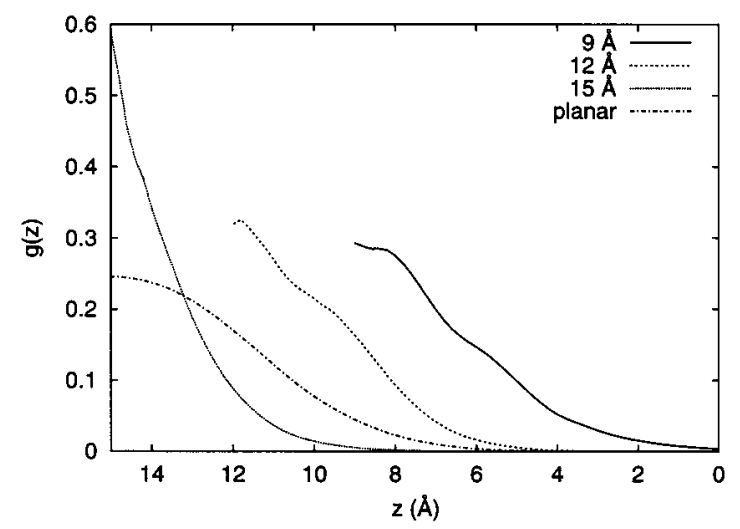

Figure 11. Distribution function for an OPLS chloride ion solvated by nonpolarizable TIP4P water molecules, located beneath interfaces of various curvatures. Curves shown are for clusters of radius $9 \AA$ (solid line), $12 \AA$ (dashed line), and $15 \AA$ (dotted line) and for a lamellar slab of thickness $30 \AA$ (dot-dashed line). The solvation depth axis is reversed to assist comparison with Figures 1-4.

The geometric model used here is fairly crude, and involves several approximations. These include the assumption that the solvent structure in the cluster is the same as it is in the bulk. The structure imposed by the chloride ion and by solventsolvent interactions is accounted for in the distance-dependent relative solvation energy $\Delta E(r)$, but there is no mechanism to account for the additional structuring effects of the interface. In addition, it is assumed that the solvent density drops from the bulk value sharply to zero at the cluster boundary. Figures 1 through 4 clearly show that this is not the case. The interface is also assumed to have uniform curvature, whereas simulations indicate that the chloride ion can induce rather significant deformations in the surface structure. Furthermore, the interface is assumed to be static, rather than the dynamically fluctuating boundary that is actually observed. And the model is not able to account for any entropic contributions associated with ioninduced solvent reorganization. None of these assumptions is true, and yet this very approximate model gives a suprisingly complete picture of the anomalous solvation behavior observed in the molecular simulations. This can be taken to indicate that the solvation behavior is largely due to the few simplistic effects that are included in the model: the dependence of solvent energy and structure on distance from the ionic solute, and the average geometric structure of the interface.

The success of this simple model is encouraging, and suggests that a similar analysis could be used successfully in related systems. The most useful benefit of this approach is that a single simulation of the bulk solution can provide all of the simulation data needed to arrive at an estimate of the solvation structure beneath an interface. As long as a system is large enough that surface effects do not perturb the bulk $\Delta E(r)$ curve too much (no fewer than 100 molecules), results can be obtained analytically at interfaces of arbitrary geometry and size, without performing additional MD simulations. This is particularly useful for very large systems, where simulation time would be prohibitive. In particular, this method could prove useful in calculating solvation distributions at structured interfaces such as microelectrodes.

Although the surface solvation phenomenon is beginning to be understood, many questions remain. For the chloride ion, the surface solvation results from a rather subtle energy balance between the different solvation shells. Is this phenomenon peculiar to chloride, or does it occur more generally? Experiments indicate that surface solvation is observed for larger halides, but the question remains unanswered for more complex anions. If anions exhibit surface solvation for surface geometries, but cations do not, this will have important implications for the ionic double layer and the electric fields present at curved interfaces. This has potential implications in electrochemistry and aqueous atmospheric chemistry (although atmospheric water droplets typically do not have radii small enough to show surface solvation for the chloride ion). Also unknown is the impact of surface segregation on transport phenomena such as diffusion. In fuel cells, for example, conductivity could potentially be enhanced by designing the microscopic polymer geometry to enhance surface solvation of the conductive ions, provided that lateral diffusion is not diminished when the ions are surface solvated.

Acknowledgment. This work was funded by the National Institutes of Health, the National Science Foundation, and the Maui High Performance Computing Center.

\section{References and Notes}

(1) Perera, L.; Berkowitz, M. L. J. Chem. Phys. 1991, 95, 1954

(2) Perera, L.; Berkowitz, M. L. J. Chem. Phys. 1992, 96, 8288.

(3) Perera, L.; Berkowitz, M. L. J. Chem. Phys. 1993, 99, 4236.

(4) Stuart, S. J.; Berne, B. J. J. Phys. Chem. 1996, 100, 11934. 1475.

(5) Arshadi, M.; Yamdagni, R.; Kebarle, P. J. Phys. Chem. 1970, 74

(6) Hiraoka, K.; Misuze, S.; Yamabe, S. J. Phys. Chem. 1988, 92, 3943.

(7) Markovich, G.; Giniger, R.; Levin, M.; Cheshnovsky, O. J. Chem. Phys. 1991, 95, 9416.

(8) Markovich, G.; Pollack, S.; Giniger, R.; Cheshnovsky, O. J. Chem. Phys. 1994, 101, 9344.

(9) Choi, J.-H.; Kuwata, K. T.; Cao, Y.-B.; Okumura, M. J. Phys. Chem. A 1998, 102, 503.

(10) Ayotte, P.; Bailey, C. G.; Weddle, G. H.; Johnson, M. A. J. Phys. Chem. A 1998, 102, 3067.

(11) Ayotte, P.; Bailey, C. G.; Weddle, G. H.; Johnson, M. A. J. Phys. Chem. A 1999, 103, 443.

(12) Perera, L.; Berkowitz, M. L. J. Chem. Phys. 1993, 99, 4222.

(13) Yeh, I.-C.; Perera, L.; Berkowitz, M. L. Chem. Phys. Lett. 1997, 264,31 .

(14) Perera, L.; Berkowitz, M. J. Chem. Phys. 1994, 100, 3085.

(15) Sremaniak, L. S.; Perera, L.; Berkowitz, M. L. J. Phys. Chem. 1996, $100,1350$.

(16) Jorgensen, W. L.; Severance, D. L. J. Chem. Phys. 1993, 99, 4233.

(17) Dang, L. X.; Smith, D. E. J. Chem. Phys. 1993, 99, 6950.

(18) Cabarcos, O. M.; Weinheimer, C. J.; Martinez, T. J.; Lisy, J. M. J. Chem. Phys. 1999, 110, 9516.

(19) Randles, J. E. B. Phys. Chem. Liq. 1977, 7, 107.

(20) Wilson, M. A.; Pohorille, A. J. Chem. Phys. 1991, 95, 6005.

(21) Jorgensen, W. L.; Chandrasekhar, J.; Madura, J. D.; Impey, R. W.; Klein, M. L. J. Chem. Phys. 1983, 79, 926.

(22) Rick, S. W.; Stuart, S. J.; Berne, B. J. J. Chem. Phys. 1994, 101, 6141.

(23) Rappé, A. K.; Goddard, W. A., III. J. Phys. Chem. 1991, 95, 3358.

(24) Hoye, J.; Stell, G. J. Chem. Phys. 1980, 73, 461.

(25) Pratt, L. R. Mol. Phys. 1980, 40, 347.

(26) Tuckerman, M.; Berne, B. J.; Martyna, G. J. J. Chem. Phys. 1992, 97, 1990.

(27) Stuart, S. J.; Zhou, R.; Berne, B. J. J. Chem. Phys. 1996, 105, 1426.

(28) Ewald, P. Ann. Phys. 1921, 64, 253.

(29) Heyes, D. M. J. Chem. Phys. 1981, 74, 1924

(30) Halley, J. W.; Hautman, J.; Rahman, A.; Rhee, Y.-J. Phys. Rev. B 1989, 40,36

(31) Hautman, J.; Klein, M. Mol. Sim. 1992, 75, 375.

(32) Onsager, L.; Samaras, N. N. T. J. Chem. Phys. 1934, 2, 528.

(33) Marcus, Y. Ion Solvation; John Wiley \& Sons: New York, 1985.

(34) Hiraoka, K.; Mizuse, S. Chem. Phys. 1987, 118, 457. 2703.

(35) Curtiss, L. A.; Frurip, D. J.; Blander, M. J. Chem. Phys. 1979, 71,

(36) Frank, H. S.; Wen, W.-Y. Discuss. Faraday Soc. 1957, 24, 133.

(37) Kusalik, P. G.; Svishchev, I. M. Science 1994, 265, 1219.

(38) Chandrasekhar, J.; Spellmeyer, D. C.; Jorgensen, W. L. J. Am. Chem. Soc. 1984, 106, 903. 